\title{
Multimodal Treatment Approach to Advanced Female Urethral Squamous Cell Carcinoma"
}

\author{
Lindsay L. Hertzig ${ }^{1}$, Richard C. Choo ${ }^{2}$, Deborah J. Lightner ${ }^{1}$, R. Jeffrey Karnes ${ }^{1 \#}$ \\ ${ }^{1}$ Department of Urology, Mayo Clinic, Rochester, USA \\ ${ }^{2}$ Department of Radiation Oncology, Mayo Clinic, Rochester, USA \\ Email: "karnes.r@mayo.edu
}

Received June 15, 2012; revised July 11, 2012; accepted July 20, 2012

\begin{abstract}
Introduction and Objectives: Primary squamous cell carcinoma of the female urethra is rare. Given the small number of cases seen, no uniform regimen for optimal treatment has been identified. We set out to review the outcomes of women with advanced urethral squamous cell carcinoma treated with a multimodality approach in order to gain insight into the optimal treatment of this rare malignancy. Methods: We identified five women with advanced urethral squamous cell carcinoma treated by a single surgeon (RJK). After obtaining IRB approval, a retrospective chart review was performed and treatment strategies and outcomes were analyzed. Results: We report on 5 cases of squamous cell carcinoma of the female urethra treated utilizing a multimodal approach. Surgical therapy included anterior exenteration in two patients, partial urethrectomy in two patients and transurethral resection in one. Patients were treated with radiation in a neoadjuvant ( 2 cases), adjuvant ( 2 cases), or definitive ( 1 case) fashion. Four of five patients received chemotherapy. With a mean follow-up of 39 months (range from 3 to 50), 4 patients remain without evidence of disease while one patient developed lung metastases 33 months after treatment. None had a locoregional tumor recurrence. Conclusion: Multi-modality approach combining surgery, radiotherapy, and chemotherapy can be effective therapeutic intervention for locally advanced, squamous cell carcinoma of the female urethra. For patients who have a significant surgical risk or desire to pursue an organ-preservation strategy, a trimodality approach of limited surgical excision combined with radiotherapy plus chemotherapy may be a reasonable alternative to anterior pelvic exenteration.
\end{abstract}

Keywords: Squamous Cell Carcinoma; Urethral Carcinoma

\section{Introduction}

Primary squamous cell carcinoma (SCC) of the female urethra is rare, representing only $0.02 \%$ of all cancers found in women [1]. Given the small number of cases treated at individual institutions, no uniform regimen for optimal treatment has been determined. We report on 5 patients treated at our institution by a single surgeon, utilizing multimodal treatment strategies. Specific treatment strategies were individualized based on patient age, tumor stage, location and lymph node involvement.

\section{Case Series}

\subsection{Case 1}

A 58-year-old female presented with acute urinary retention. An abnormal physical exam (fullness of the anterior vagina) prompted transurethral and transvaginal biopsies revealing SCC (invasive grade 3 of 4). MRI was com-

${ }^{*}$ Conflicts of interest: None to disclose.
${ }^{\#}$ Corresponding author. pleted and showed a bulky mass $(4.7 \times 4.0 \times 4.5 \mathrm{~cm})$ within the pelvis extending from the base of the bladder to the perineum. No obvious pelvic or inguinal lymphadenopathy was seen and no evidence for distant metastasis was identified. With bladder neck involvement (also visualized on cystoscopy), the patient had a clinical stage T3N0 tumor.

Given a borderline resectability of her tumor, a multimodality therapeutic approach was planned. The patient started with neoadjuvant radiotherapy plus chemotherapy (as a radiosensitizer: cisplatin at a dose of $30 \mathrm{mg} / \mathrm{m}^{2}$ weekly for 5 weeks). Radiotherapy consisted of 5000 cGy to the pelvic mass and 4375 cGy to the bilateral inguinal and pelvic lymph nodes over 25 fractions in 5 weeks, using IMRT (intensity modulated radiation therapy) technique. Of note, the patient's urinary retention resolved approximately 2 weeks after starting this neoadjuvant therapy.

MR imaging confirmed the clinical impression of significant down-staging (Figure 1), and the patient was treated with an uncomplicated anterior exenteration, an 


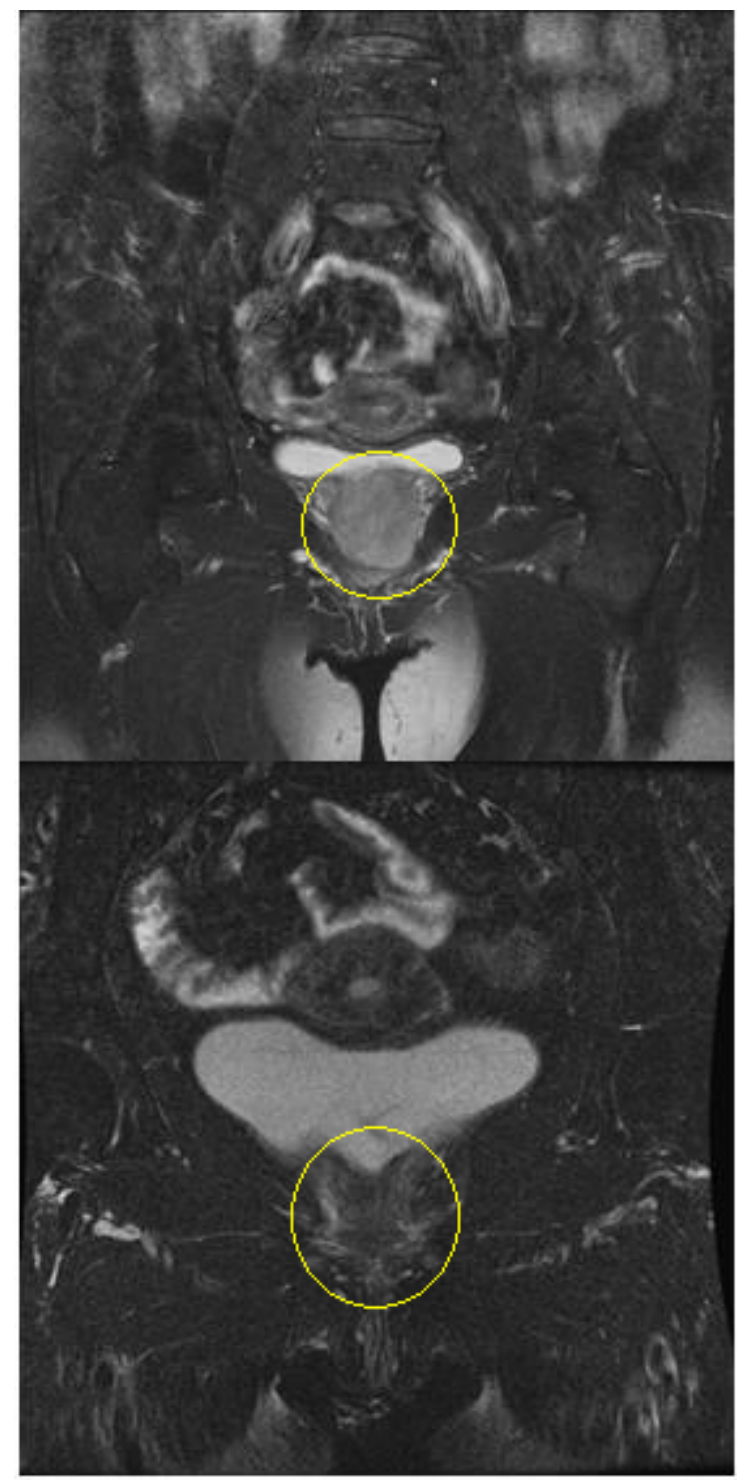

Figure 1. Coronal T1 MRI. Prior to chemoradiation (top). Post chemoradiation (bottom). The urethral mass reduced in size from $4.7 \times 4.0 \times 4.5 \mathrm{~cm}$ to $4.7 \times 2.1 \times 1.0 \mathrm{~cm}$.

extended pelvic lymph node dissection, and Indiana pouch creation. Pathologic evaluation of the surgical specimen showed no residual malignancy (i.e. pT0N0). At the initial 4-month follow-up, the patient remained free of tumor recurrence.

\subsection{Case 2}

A-56-year old female five years disease free from cervical dysplasia (status post vaginal hysterectomy), vaginal intraepithelial neoplasia (VAIN) and vulvar dysplasia (VIN) (status post excisional biopsy, laser ablation and topical therapy) noted a perineal mass and hematuria.

A submucosal $2.7 \mathrm{~cm}$ mass was identified along the anterior vaginal wall and confirmed as Grade 3 (of 4) invasive squamous cell carcinoma on cystoscopic biopsy of the mid urethra. MRI showed a mass-like thickening of the urethra extending from the introitus to the bladder lumen and abutting the pubic bone. Clinically, this was stage T3 disease with involvement of the bladder neck.

A multimodality therapeutic intervention included neoadjuvant radiotherapy plus chemotherapy (carboplatin at a dose of AUC 2 and Taxol $50 \mathrm{mg} / \mathrm{m}^{2}$ weekly for 6 weeks). Her radiotherapy comprised of $4500 \mathrm{cGy}$ to the pelvis including the primary tumor and pelvic lymph nodes, and 4025 cGy to the bilateral inguinal lymph nodes. It was delivered over 25 fractions in 5 weeks, using 3-dimensional conformal radiation therapy technique. Following the completion of radiotherapy, the patient received 2 additional cycles of carboplatin and Taxol.

MRI restaging showed a $50 \%$ decrease in tumor size to $1.3 \mathrm{~cm}$. The patient then underwent a planned anterior pelvic exenteration, extended pelvic and superficial inguinal lymphadenectomy and ileal conduit urinary diversion. Pathology revealed no residual tumor (pT0N0).

At 49 months of follow up, the patient remains without evidence of recurrent disease. Also, of note, the patient has had no recurrence of VAIN or VIN.

\subsection{Case 3}

A 78-year-old female with a long-standing history of irritative voiding symptoms developed acute urinary retention. A urethral biopsy was performed revealing SCC and she was treated with distal urethrectomy, with squamous carcinoma in situ (CIS) in the final pathology. Surveillance cystoscopy revealed recurrent suspicious urethral lesions, and the patient presented to our institution. CT of the abdomen and pelvis was performed revealing no evidence for metastatic disease. Pelvic MRI identified a urethral lesion $1.3 \mathrm{~cm}$ in diameter and $3.4 \mathrm{~cm}$ in length. Also noted was a $1.2 \mathrm{~cm}$ enhancing left inguinal lymph node.

The patient proceeded with a cystoscopic biopsy, which revealed proximal urethral SCC. Because the patient decided against an aggressive surgery, but was desirous of local tumor control, maximal surgical tumor debulking by transurethral resection, with organ-preservation was planned to be followed by radiotherapy + chemotherapy. Final pathology revealed invasive grade 3 (of 4) squamous cell carcinoma (pT2), for clinical stage III disease (T2N1).

She was treated with definitive radiotherapy, 6150 cGy to the primary tumor, 5400 cGy to the left inguinal lymphadenopathy, and 5100 cGy to the right groin and pelvis in 30 fractions, using IMRT technique. In addition, 6 weekly concurrent cisplatin infusions were planned at a dose of $25 \mathrm{mg} / \mathrm{m} \mathrm{sq}$ as a radiosensitizer. The patient, however, tolerated the chemotherapy poorly, completing only one dose due to nausea, diarrhea and secondary or- 
thostasis requiring hospitalization.

Restaging MRI and cystoscopy showed no residual urethral disease and no pelvic or inguinal lymphadenopathy. Approximately 33 months following the completion of radiotherapy, on routine follow-up imaging, the patient developed bilateral pulmonary nodules confirmed on biopsy as metastatic SCC. She was able to complete palliative chemotherapy in the form of carboplatin and Taxol. At 44 months after initial treatment, she remains alive without evidence of locoregional recurrence.

\subsection{Case 4}

A 51-year old female presented with vaginal spotting and vulvar irritation. Biopsy was performed revealing CIS of the labia majora, which extended to the urethral meatus. Excisional biopsy and laser vaporization were completed. Pathology from the biopsy revealed VIN III. The patient continued to have recurrent dysplasia in the periurethral area and was referred for cystoscopy. At the time, the patient reported symptoms or urinary obstruction as well as blood spotting.

Clinical examination revealed a mass at the distal posterior urethra and an enlarged right groin node. Cystoscopy revealed extrinsic narrowing of the distal urethra. Excisional biopsy confirmed poorly differentiated squamous cell carcinoma (pT2).

MRI confirmed the enlarged right inguinal node (measuring $2.2 \mathrm{~cm}$ ). PET scan revealed increased metabolic activity along the right anterior wall of the distal urethra and an enlarged hypermetabolic right groin node (Figure 2).

The patient was treated with distal urethrectomy, pelvic lymphadenectomy and inguinal lymph node dissection (superficial and deep on the right, superficial only on the left). Pathologic staging confirmed poorly differentiated squamous carcinoma of the urethra, making her

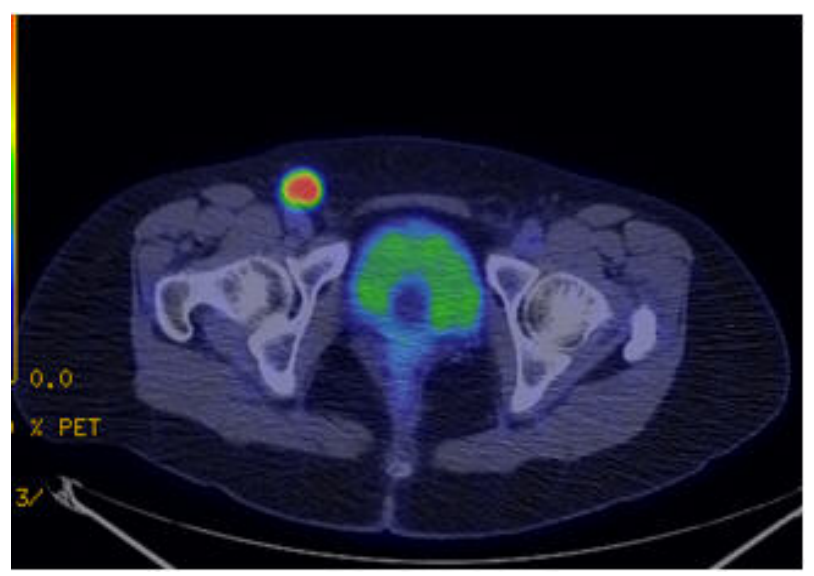

Figure 2. FDG PET/CT with hypermetabolic right inguinal lymph node.
Stage III, pT2N1. Her urethral margins were negative with a single positive metastatic lymph node without extracapsular extension in the right groin.

The patient then underwent postoperative adjuvant radiotherapy, which delivered $6000 \mathrm{cGy}$ to the urethra and right groin and $5100 \mathrm{cGy}$ to the left groin and pelvis over 30 fractions. This was followed by adjuvant chemotherapy for which she received 3 out of 4 cycles of cisplatin and Taxol at a dose of $75 \mathrm{mg} / \mathrm{m}^{2}$. Despite dose reductions, the patient experienced significant chemotherapy-related side effects requiring hospitalization, including severe nausea, dehydration and neutropenia.

With 50 months of follow up since the multi-modality therapy, the patient remains without evidence of recurrent disease.

\subsection{Case 5}

A 55-year-old female presented with urethral bleeding and a palpable perineal mass. Exam confirmed a firm 1.3 $\mathrm{cm}$ mass at the urethral meatus, cystoscopy showed no other urethral or bladder lesions. MRI revealed a $1.3 \mathrm{~cm}$ periurethral mass and at $1.2 \mathrm{~cm}$ right inguinal lymph node. Nodal status could not be assessed clinically due to her obesity.

Excisional biopsy and pathology showed an invasive, high grade carcinoma, not able to be differentiated as squamous or urothelial in origin.

PET scan confirmed the hypermetabolic enlarged right inguinal node. A formal distal urethrectomy confirmed the diagnosis of grade 3 pT2 squamous cell carcinoma with negative margins. The bilateral inguinal lymph node dissection (superficial and deep on the right, superficial only on the left) confirmed one right superficial node positive for metastatic grade $3 \mathrm{SCC}$, making this patient Stage III, pT2, N1.

The patient then underwent postoperative radiotherapy, which delivered $5850 \mathrm{cGy}$ to the urethra and right groin and $5100 \mathrm{cGy}$ to the left groin and pelvis in 30 fractions. Chemotherapy was foregone due to concerns with skin and wound issues. At 4-month post-radiotherapy, she developed skin breakdown and soft tissue necrosis in the right groin region. This was successfully treated with hyperbaric oxygen therapy.

With 50 months of follow up, the patient remains free from recurrent disease.

\section{Discussion}

Primary urethral squamous cell carcinoma in the female is rare. The National Cancer Institute Surveillance, Epidemiology and End Results (SEER) database reported an overall annual incidence of SCC in women to be 1.5 cases per million. This increased to 2.9 cases per million for the 75 to 84 year-old age group [2]. Given the rarity 
of this tumor, most information regarding treatment is based on retrospective reviews in which varying treatment modalities are utilized.

Determining the size and location of the tumor as well as the TNM stage is essential in treatment planning. Dalbagni and colleagues [3] showed that primary stage, nodal status and site of disease were independent predictors of survival in 72 patients. For low-stage lesions, single modality therapy in the form of radiation or surgery may provide a good chance for disease control and minimize morbidity. Many patients, however, present with advanced stage disease and multiple investigators have reported on the inadequacies of single modality therapy in this situation.

Surgical treatment alone for advanced disease has resulted in survival rates of $20 \%-30 \%$ at 5 years [4]. For single modality radiation therapy, Garden et al. [5], reported in a large series of 97 women that the 5- and 10 -year actuarial survival rates were $41 \%$ and $31 \%$, respectively with a local control rate of $64 \%$. Foens et al. [6] compared the utility of radiation to surgery in female urethral carcinoma and noted that while radiation may have a superior local control rate (64\% vs. $40 \%)$; 5-year survival rates were similar. Narayan and Konety [7] examined a combined approach of radiation and surgery and reported 55\% survival in patients with advanced disease treated with this combined approach compared to $34 \%$ survival in patients treated with radiation alone.

Chemoradiation has been successful for the treatment of advanced urethral (SCC) as reported in several small case series [8-13] suggesting that optimal local and distant control will involve a combination of chemotherapy, radiation and surgery $[3,4]$.

In this series, we present five cases of advanced SCC of the urethra managed with a multimodality therapy; two with advanced local disease and three with nodal metastases. All achieved locoregional tumor control and remained alive at the time of last follow-up. One with inguinal nodal metastasis at presentation developed lung metastasis at 33 months follow-up.

The two patients presenting with locally advanced disease (clinical stage T3N0) were treated with neoadjuvant radiation with concurrent weekly chemotherapy (as a radiosensitizer; cisplatin in one and carboplatin and Taxol in the other). They both subsequently underwent consolidative surgery with anterior exenteration and were found to have no residual disease at the time. Both are alive and free of disease recurrence at 4 and 49 months respectively. These cases are illustrative of the radiosensitivity of squamous cell carcinoma of the female urethra, similar to squamous cell carcinoma of the vulva or the penis, and that chemotherapy may enhance the efficacy of radiotherapy. This is consistent with Gheiler et al. [4] who reported that only one of eight patients in their se- ries had residual disease at the time of surgery after undergoing neoadjuvant chemoradiation. Unlike this report, the Gheiler case series included mixed urethral cancer histology and both male and female patients.

Although the need for consolidative surgery in this setting remains unclear, this case series demonstrates that pre-operative radiotherapy can be cytoreductive, improving resectability in bulky tumors. In these cases, the younger otherwise healthy patients (ages 58 and 56) were treated with the most aggressive treatment approach in order to limit the possibility of local recurrence and secondary distant metastasis.

In some cases, local surgical treatment directed at organ-sparing may be appropriate. Our third case of invasive $\mathrm{T} 2 \mathrm{~N} 1$ disease in a 78 year old woman was managed with an organ-preservation strategy using a trimodality therapy, consisting of a maximal local tumor debulking followed by definitive radiotherapy plus chemotherapy. Achieving local tumor control and debulking, prior to radiotherapy is well established for other genitourinary malignancies such as advanced urothelial carcinoma of the bladder. While this patient subsequently developed lung metastases, she remains free from locoregional recurrence. This case illustrates that a patient presenting with nodal metastasis is at risk of distant metastasis even if locoregional tumor control is achieved, and that chemotherapy may be able to sterilize micrometastatic disease.

Our final two patients had urethral lesions amenable to partial urethrectomy with negative surgical margins. This limited surgery allowed both patients to maintain normal urinary continence, which is a significant advantage over anterior exenteration requiring a urinary diversion. Both, however had clinically evident nodal disease and underwent groin dissections confirming metastasis with complete resections. In both patients, postoperative adjuvant radiotherapy with or without chemotherapy was added to minimize the risk of locoregional tumor recurrence. Both patients are alive and free from disease recurrence at 50 months follow-up. Theses cases suggest that in a selected case, a combination of limited surgical excision plus postoperative radiotherapy, as an alternative to anterior exenteration, may be an efficacious treatment and allows normal voiding function.

Lastly, in locally extensive disease or those with adverse pathologic features such as extracapsular extension in a metastatically involved node or multiple nodal metastases, it is prudent to offer postoperative radiotherapy and/or chemotherapy to minimize the risk of locoregional tumor recurrence and distant metastasis as illustrated by our cases.

\section{Conclusion}

Urethral carcinoma of the female urethra is rare and mul- 
tiple treatment regimens have been reported. Many patients (as those reported in our series) present with advanced disease. For locally advanced squamous cell carcinoma of the female urethra, a multi-modality approach combining surgery, radiotherapy, and chemotherapy can be effective therapeutic intervention. For patients who have a significant surgical risk or desire to pursue an organ-preservation strategy, a trimodality approach of limited surgical excision combined with radiotherapy plus chemotherapy may be considered as an alternative to anterior pelvic exenteration.

\section{REFERENCES}

[1] D. E. Johnson and J. R. O'Connell, "Primary Carcinoma of the Female Urethra," Urology, Vol. 21, No. 1, 1983, pp. 42-45. doi:10.1016/0090-4295(83)90120-6

[2] M. A. Swartz, M. P. Porter, D. W. Lin, et al., "Incidence of Primary Urethral Carcinoma in the United States," Urology, Vol. 68, No. 6, 2006, pp. 1164-1168. doi:10.1016/j.urology.2006.08.1057

[3] G. Dalbagni, Z. F. Ahang, L. Lacombe, et al., "Female Urethral Carcinoma: An Analysis of Treatment Outcome and a Plea for a Standardized Management Strategy," British Journal of Urology, Vol. 82, No. 6, 1998, pp. 835841. doi:10.1046/j.1464-410X.1998.00878.x

[4] E. L. Gheiler, M. V. Tefilli, et al., "Management of Primary Urethral Cancer," Urology, Vol. 52, No. 3, 1998, pp. 487-493. doi:10.1016/S0090-4295(98)00199-X

[5] A. S. Garden, G. K. Zagars and L. Declos, "Primary Carcinoma of the Female Urethra: Results of Radiation Therapy," Cancer, Vol. 71, No. 10, 1993, pp. 3102-3108. doi:10.1002/1097-0142(19930515)71:10<3102::AID-CN CR2820711034>3.0.CO;2-2

[6] C. S. Foens, D. H. Hussey, J. J. Staples, et al., "A Comparison of the Roles of Surgery and Radiation Therapy in the Management of Carcinoma of the Female Urethra," International Journal of Radiation Oncology, Biology, Physics, Vol. 21, No. 4, 1991, pp. 961-968. doi:10.1016/0360-3016(91)90736-N

[7] P. Nayaran and B. Konety, "Surgical Treatment of Female Urethral Carcinoma," Urologic Clinics of North America, Vol. 19, No. 2, 1992, pp. 373-382.

[8] I. Hara, S. Hikosaka, H. Eto, et al., "Successful Treatment for Squamous Cell Carcinoma of the Female Urethra with Combined Radio and Chemotherapy," International Journal of Urology, Vol. 11, No. 8, 2004, pp. 678-682. doi:10.1111/j.1442-2042.2004.00859.x

[9] D. W. Johnson, et al., "Low Dose Combined Chemotherapy/Radiotherapy in the Management of Locally Advanced Urethral Squamous Cell Carcinoma," The Journal of Urology, Vol. 141, No. 3, 1989, pp. 615-616.

[10] M. R. Licht, et al., "Combination Radiation and Chemotherapy for the Treatment of Squamous Cell Carcinoma of the Male and Female Urethra," The Journal of Urology, Vol. 153, No. 6, 1995, pp. 1918-1920. doi:10.1016/S0022-5347(01)67354-3

[11] S. Nicholson, D. Tsang and D. Summerton, "Aggressive Combined-Modality Therapy for Squamous Cell Carcinoma of the Female Urethra," Nature Clinical Practice Urology, Vol. 5, No. 10, 2008, pp. 574-577. doi:10.1038/ncpuro1211

[12] A. B. Shah, et al., "Squamous Cell Cancer of the Female Urethra: Successful Treatment with Chemoradiotherapy," Urology, Vol. 25, No. 3, 1985, pp. 284-286. doi:10.1016/0090-4295(85)90329-2

[13] L. N. Tran, et al., "Combination Chemotherapy and Radiotherapy for a Locally Advanced Squamous Cell Carcinoma of the Urethra: A Case Report," The Journal of Urology, Vol. 153, No. 2, 1995, pp. 422-423. doi:10.1097/00005392-199502000-00042 